\title{
Discrimination Among Different Lemon Juice Samples Using Chronoamperometry in a Microfluidic Channel
}

\author{
Asma Souri, Hasti Sardari, Faramarz Hossein-Babaei \\ Electronic Materials Laboratory, Electrical Engineering Department, K. N. Toosi University of \\ Technology, Tehran, 16317-14191, Iran \\ asmasouri@yahoo.com
}

\begin{abstract}
Food quality control has always been an issue for both the food producer and consumer. The dominant trend in analyses of fluids is miniaturization based on microfluidics concepts. Here, we demonstrate discriminating between different lemon juice samples by performing a chronoamperometry in a microfluidic channel. A droplet of lemon juice is injected into a microfluidic channel and a voltage waveform is applied on the channel from a preprogramed power supply. The temporal variations of the electric current passing through the channel is recorded, digitized and stored. The recorded data is used for defining a feature vector for each sample. Feature vectors for different samples are classified into different classes using PCA. The system is able to detect and recognize the sample type in less than 5 minutes.
\end{abstract}

Key words: Electronic tongue, microfluidics, food quality, chronoamperometry, electrolyte

\section{Introduction}

The sharp increase in incidences of food poisoning reflects the need for use of food quality assessment systems such as electronic tongues. Extracting analytical information from lemon juice samples injected into microfluidic channels facilitate better detection and discrimination among them [1, 2]. A disturbingly large percentage of the commercially available lemon juice is diluted with cheaper substances, demanding a more accurate system to detect and recognize such additives; necessitating rapid, simple, inexpensive methods for food quality assessments.

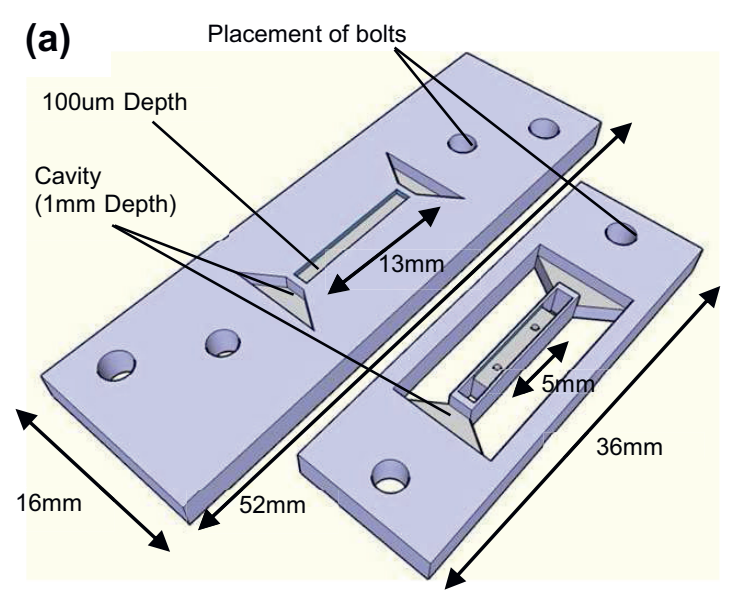

Here, we present a novel electronic tongue based on chronoamperometry in a microfluidic channel to discriminate among different beverage samples; the acquired data is utilized for the classification of the commercially available lemon juice samples into different quality-based classes.

\section{Methods}

The structure of the microfluidic channel system is schematically shown in Fig. $1 \mathrm{a}$ and b. The channel system is fabricated on a PMMA chip by $\mathrm{CO}_{2}$ laser engraving [3-5].

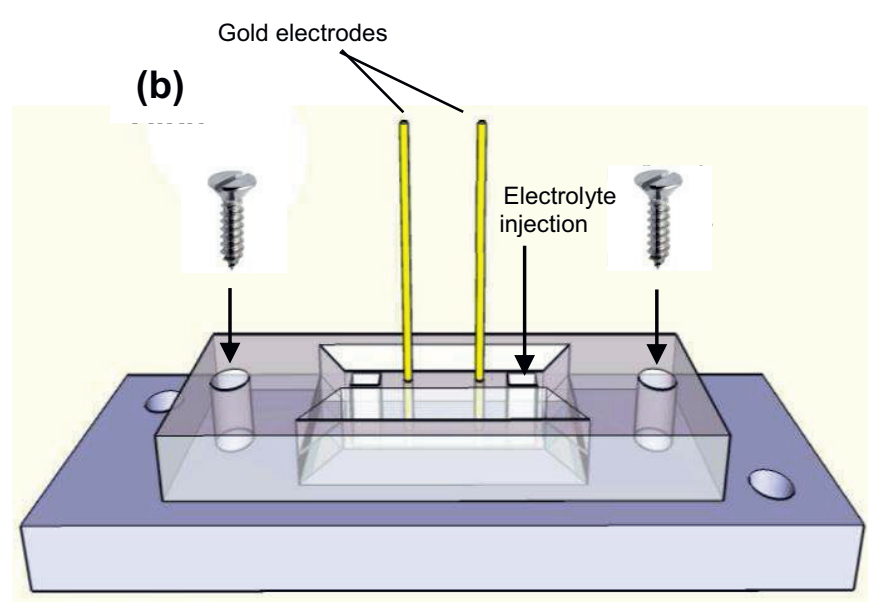

Fig. 1. (a) and (b), Schematic view of a fabricated device; PMMA components (a), assembly (b). 
Two $0.6 \mathrm{~mm}$ diameter gold electrodes are laterally inserted into the channel via two holes devised on the channel wall as shown in Fig. 1. After injecting the analyte into the channel, the voltage waveform shown in Fig. 2 is applied to the electrodes and the voltage on the series resistor (see Fig. 3) is recorded. This voltage is proportional to electric current passing through the channel. A sample of the recorded temporal profiles obtained for different lemon juice samples are given in Fig. 2.

Three different lemon juice samples are tested along with a sample of the natural lemon juice freshly extracted in our laboratory.

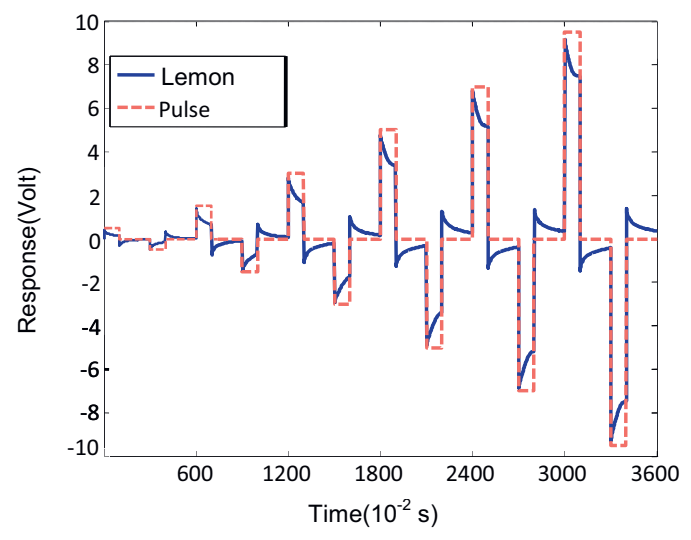

Fig. 2. Voltage waveform applied to the microchannel and the recorded response profile.

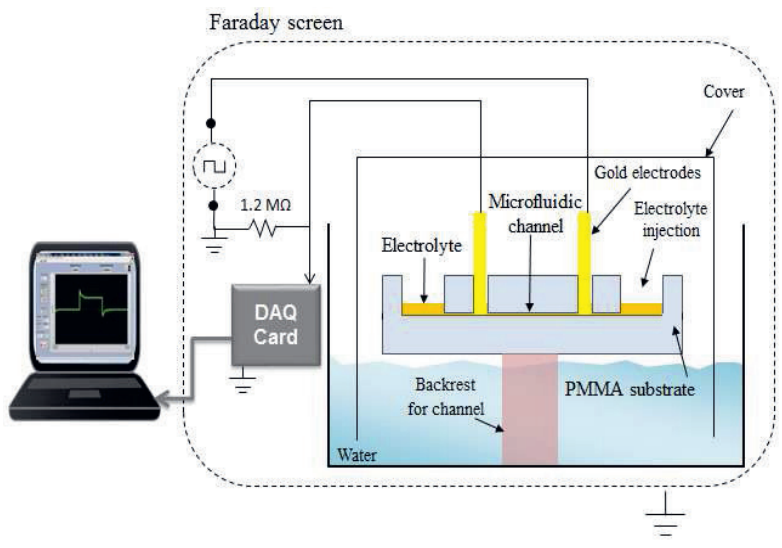

Fig. 3. Schematics of the experimental setup.

\section{Results}

A 300-dimensional feature vector is constructed for each sample based on its respective temporal response. The feature collection is carried out heuristically based on the most dissimilar regions of the recorded responses. Applying principle component analysis (PCA) reduces the feature space dimensions from 300 to 3 , allowing visualization in a 3-dimensional feature space. According to Fig. 4, a good discrimination between the natural lemon and the 3 commercial samples are achieved.

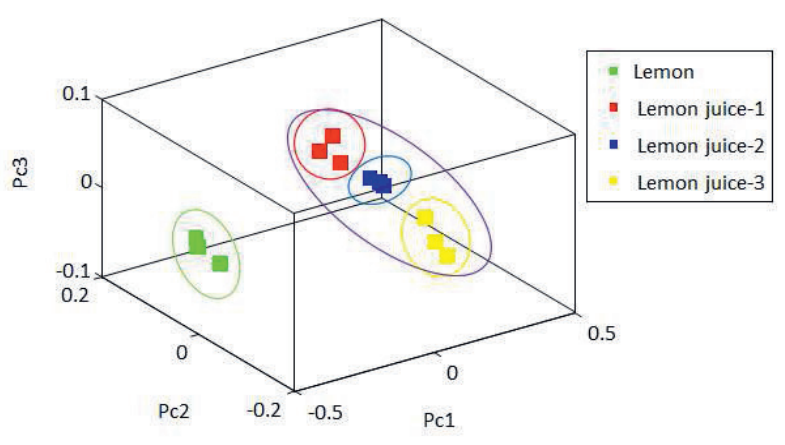

Fig. 4. Successful classification of the lemon juice samples in the feature space.

\section{Conclusion}

A simple and fast method was presented for performing chronoamperometry on various fluidic analytes in a microchannel. It was shown that the information acquired by chronoamperometry in a microfluidic channel is sufficient for distinguishing between different beverages. The method uses only $8 \mu \mathrm{L}$ of the sample and is a useful microfluidic system for fast assessments in the food industry.

\section{References}

[1] Hossein-Babaei, Faramarz, and Ali Hooshyar Zare. "The selective flow of volatile organic compounds in conductive polymer-coated microchannels." Scientific Reports 7 (2017): 42299; doi:10.1038/srep42299

[2] Hossein-Babaei, Faramarz, and Kianoosh Nemati. "A concept of microfluidic electronic tongue." Microfluidics and nanofluidics 13.2 (2012): 331-344. doi: 10.1007/s10404-0120967-X

[3] Hossein-Babaei, Faramarz, Mohammad Paknahad, and Vahid Ghafarinia. "A miniature gas analyzer made by integrating a chemoresistor with a microchannel." Lab on a Chip 12.10 (2012): 1874-1880. doi: 10.1039/C2LC00035K

[4] Hossein-Babaei,Faramarz, Ali Hooshyar Zare, and Vahid Ghafarinia, and Sobhan Erfantalab, "Identifying volatile organic compounds by determining their diffusion and surface adsorption parameters in microfluidic channels," Sensors and Actuators B: Chemical, 220 (2015):607-613; doi: 10.1016/j.snb.2015.06.014.

[5] Hossein-Babaei, Faramarz and Ghafarinia. Vahid, "Gas analysis by monitoring molecular diffusion in a microfluidic channel", Analytical Chemistry, 82 (2010): 8349-8355; doi: 10.1021/ac101767r 